\title{
Respiratory Malfunction Arising from Mental Factor
}

National Cancer Institute

\section{Source}

National Cancer Institute. Respiratory Malfunction Arising from Mental Factor. NCI

Thesaurus. Code C35390.

A respiratory malfunction for which mental factors play a causative role. 